\title{
A Regional Protection Partition Strategy Considering Communication Constraints and Its Implementation Techniques
}

\author{
Zhenxing $\mathrm{Li}^{1}{ }^{1}$, Yang Gong ${ }^{1, *}$, Lu Wang ${ }^{1}$, Hong Tan ${ }^{2}$, Prominent Lovet Kativu ${ }^{1}$ and \\ Pengfei Wang ${ }^{1}$ \\ 1 College of Electrical Engineering and New Energy, China Three Gorges University, Yichang 443000, China; \\ lizhenxing@ctgu.edu.cn (Z.L.); 2012909204@ctgu.edu.cn (L.W.); 2016110508137@ctgu.edu.cn (P.L.K.); \\ seasky@ctgu.edu.cn (P.W.) \\ 2 School of Electrical Engineering, Chongqing University, Chongqing 400044, China; 20181101035@cqu.edu.cn \\ * Correspondence: 2012109424@ctgu.edu.cn; Tel.: +86-176-7132-0620
}

Received: 13 August 2018; Accepted: 18 September 2018; Published: 20 September 2018

check for updates

\begin{abstract}
Regional protection based on multisource information of a regional power network depends on communication technology. A partition strategy considering communication constraints and implementation techniques must be considered to realize the regional protection of a large power grid. This paper aims at examining the technical requirements of rapid and reliable regional protection, considering the number of hops as the factor affecting communication between secondary substations and primary substations, and combining this with the equalization of substations. Then, a primary substation selection model of regional protection based on an exhaustive method is proposed using the Floyd-Warshall algorithm (an algorithm for finding shortest paths in a weighted graph). The partition model is further established according to the multifactors that affect the communication delay time for regional protection. Focusing on the N-1 channel fault in the preset region after the formation of the subregions, this paper analyzes the circuitous process of information in an interrupt channel and considers the influence of communication delay time to further improve the partition strategy. Finally, this paper puts forward techniques for partition strategy implementation based on graph theory; an example analysis of an actual power network is also given, and the conclusions of multiple partitions of the same power network are compared and analyzed. Besides this, partition suggestions and theoretical guidance considering actual engineering demands are given.
\end{abstract}

Keywords: regional protection; communication constraint; partition strategy; graph theory

\section{Introduction}

The development of technology in this era has sped up the process of replacing traditional backup protection with regional protection. Traditional backup protection makes trip decisions through local information; this has some disadvantages such as difficulty in setting coordination and poor adaptability [1-3]. With the development of communication technology, regional protection which gathers multisource information for decision-making has been widely studied for addressing the shortcomings of traditional backup protection [4-6]. In order to achieve regional protection, a large power grid is divided into several regions, regional decision-making centers are set up, and multisource information in the region is collected by means of communication technology. Thereby, online setting of in situ protection is realized, or a new protection criterion is constructed based on multi-information discrimination [7-9]. The protection will be completely ineffective if the communication has some problem such as too long a delay, traffic in the channel, packet loss, etc. Therefore, the communication of the system is extremely important for this kind of new protection $[10,11]$. The rationality of partitioning 
directly determines the quickness and accuracy of transmitting multisource information to regional decision-making centers. Therefore, a reasonable partition strategy for the large power grid has vital significance for achieving reliable regional protection.

At present, many scholars have put forward regional protection partition ideas and implementation techniques. In reference [12], an initial primary substation was selected to divide a finite element region with a specified radius. Other primary substations were determined outside the finite element region according to the principle of primary substation selection. In reference [13], the primary substation was selected according to the qualitative principle, and a round grid search was carried out with three logical lengths as the radius. In reference [14], a regional autonomous backup protection partition scheme was proposed. The partition scheme was formulated according to the principle of a certain primary substation radius, and the partition algorithm was executed several times. The final result was determined as the scheme with fewest primary substations. In reference [15], the scholar comprehensively considered real-time, economic, and equilibrium factors to establish reference indicators, and used a genetic algorithm to perform a two-layer search to achieve partitioning. In reference [16], the scholar designated a primary substation according to geographical factors, and divided the distance from the primary substation to a region with a topological distance of less than 2. Then, some artificial optimizations were made according to the principle of optimization. Based on the adjacency matrix, the shortest path search method was used to determine the region boundary in reference [17]. Taking the boundary secondary substation as the starting point, the fuzzy comprehensive evaluation method was used to determine the location of the next primary substation. Then, the search process was repeated until the partition of the area was completed. The methods of these studies are mainly based on the electrical topology of the power network, substation geographical location, or protection principle, according to the regional finite element or grid type to divide the area. However, the partition should further consider the influence of communication constraints to make the protection regions more balanced and shorten the delay time.

The object of the partition in this paper is a regional protection communication system which takes long-term planning into account. The following are the contributions of this paper: The basic structure and how the information can be transmitted will be introduced in Section 2. Basic requirements of regional protection which must be considered when we design the strategy will also be stated in this part. The primary substation selection model, secondary substation partition model, and partition correction which formulate the strategy are presented in Section 3. The partition process will be explained in Section 4 by means of an example. Further, a real example in the eastern region of Hubei province, China, which can verify the applicability of the partition strategy to a larger system, will be tested in Section 5 . The results of the partitioning and suggestions on how to choose the optimal one will be given, and a comparison of results with those from other similar studies will be done in this part. Finally, conclusions will be drawn in Section 6.

\section{Basic Structure and Basic Requirements of Regional Protection}

Wide-area protection, hierarchical protection, system protection, and intelligent center protection all aim to improve upon traditional backup protection. These protection strategies have some similarities. One of these similarities is that the primary substation obtains the secondary substation information of the regional power network through communication technology in order to construct protections. This kind of protection is called regional protection.

The structure of regional protection can generally be categorized into distributed structure, centralized structure, and hybrid structure [18]. Several decision-making primary substations need to be set up regardless of the structure of the protection system (each secondary substation of a distributed system can be regarded as a primary substation). Every primary substation acquires the information of multiple secondary substations in its region. Then, it makes the decision of tripping based on the multi-information it receives [12]. A communication system schematic of regional protection and the implementation of regional protection information transmission are shown in Figure 1. In each 
substation, an Intelligent Electronic Device (IED) collects the electrical quantity information of the system and the status information of its installation. Then, the real-time measurement information or processed information is transmitted to the substation main control unit through the process layer bus and the substation control layer bus in the form of an SV (Sampled Value) or GOOSE (Generic Object Oriented Substation Event) message. Then, the information is uploaded to the decision-making unit of the regional primary substation through a Synchronous Digital Hierarchy (SDH) optical fiber network with the help of Asynchronous Transfer Mode (ATM) communication technology. When the system fails, the primary substation decision-making unit judges the fault elements according to the collected multisource information and formulates a protection strategy. Then, it sends trip signals to the relevant secondary substation in the form of an SV message. The relevant IED then performs the protection function. There are some secondary substations sending related measurements to other regional primary substations at the same time at the junction of regions $[19,20]$.

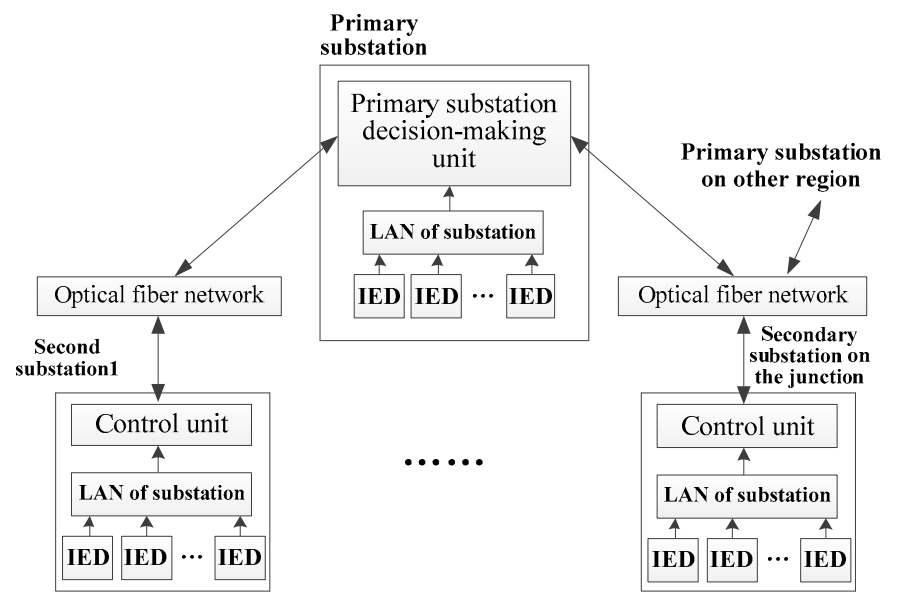

Figure 1. A communication system schematic of regional protection and the implementation of regional protection information transmission.

Some existing regional protection algorithms are based on the premise that information can be transmitted to the decision center quickly and effectively. In another word, the basic requirements of partitioning are that the communication delay is short and that channel utilizations are relatively balanced in each subregion. However, the traditional communication methods cannot meet these requirements in practice. Therefore, the communication system of a large power grid needs to be partitioned by means of a new partition strategy considering the basic requirements before regional protection is used.

\section{Protection Partition Strategy}

\subsection{Primary Substation Selection Model}

The main duty of regional protection is to realize fast identification and reliable removal of faulty components based on multisource information. Selecting the path with the shortest communication delay time to transmit the multisource information will be more favorable for the system. The influential factors of communication delay time include information transmission delay time and conversion delay time. There are two important parameters which can aid in choosing the optimal primary substation combination: one is called the minimum hops sum and the other is called the link degree sum. The optimal primary substation combination can be chosen by the Floyd-Warshall algorithm based on a model considering the above two parameters [21,22]. These two parameters will be explained by means of the example shown in Figure 2. The example has 18 substations and 27 channels. 


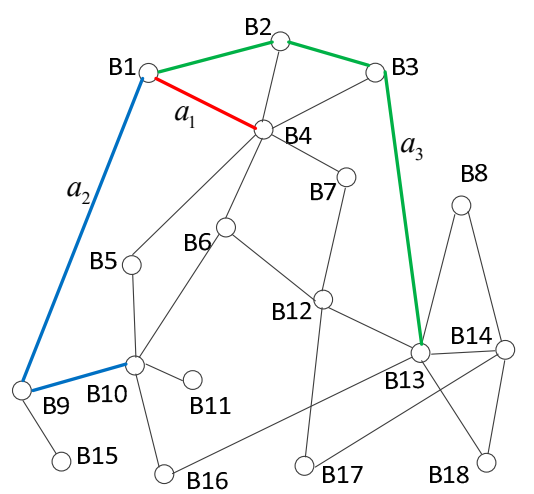

Figure 2. An example system with 18 substations and 27 channels, which can be used to explain the minimum hops sum, the link degree sum, and the primary substation selection model.

Each communication bandwidth in the regional protection system should preferably be guaranteed to be below $50 \%$ so that the channel can stay in good operation [23]. Therefore, the average number of substations in a single region can be estimated preliminarily based on the average traffic of substations. Then, the number of primary substations can be calculated. This is shown in Equation (1):

$$
\left\{\begin{array}{l}
n_{\text {avr }}=\bar{B} \times 50 \% \div \bar{M} \\
n_{\text {pri }}=n \div n_{\text {avr }}
\end{array}\right.
$$

where $n_{\text {avr }}$ is the average number of substations in each area; $\bar{B}$ is the average channel bandwidth of the system; $\bar{M}$ is the average data size of each station in the system; $n$ is the total number of substations; and $n_{p r i}$ is the number of primary substations.

In practical applications, the number of primary substations can be increased or decreased by $1 \sim 2$ on the basis of $n_{p r i}$. The respective partition results corresponding to different numbers of primary substations are obtained. Based on these partition results, the optimal partition can be selected by considering factors that must be considered in engineering practice, such as economy, communication delay, and so on. Therefore, $n_{p r i}$ is 3 for the example in Figure 2 .

In mathematics, an $x$-combination of a set $Z$ is a subset of $x$ distinct elements of $Z$. If the set has $y$-many elements, the number of $x$-combinations is equal to the binomial coefficient

$$
\mathrm{C}_{x}^{y}=\frac{y(y-1) \cdots(y-x+1)}{x(x-1) \cdots 1} .
$$

Therefore, there are $\mathrm{C}_{3}^{18}$ possible primary substation combinations $P(1), P(2), \ldots, P\left(\mathrm{C}_{3}^{18}\right)$. One of these is chosen to explain the two parameters. For example, $P(j)$ including B4, B10, B13 is selected. For secondary substation B1, the minimum hop counts between B1 and B4, B1 and B10, and B1 and $\mathrm{B} 13$ are $\mathrm{J}_{1}, \mathrm{~J}_{2}$, and $\mathrm{J}_{3}$, respectively. The minimum of $\mathrm{J}_{1}, \mathrm{~J}_{2}$, and $\mathrm{J}_{3}$ is called the minimum minimum hop count $\mathrm{s}_{\mathrm{B} 1}$. It can be acquired by using the Floyd-Warshall algorithm based on the connection matrix $A$. The method of constructing the connection matrix based on graph theory is shown in Equation (3).

$$
A(g, h)= \begin{cases}1, & g \text { and } h \text { are directly connected } \\ \text { inf }, & g \text { and } h \text { are not directly connected } \\ 0, & g=h\end{cases}
$$

In Equation (3), $g$ and $h$ are the number of substations. For example, $A(1,1), A(1,2)$, and $A(1,3)$ are equal to 0,1 , and Inf in this example, respectively. The minimum hops sum $S_{P(j)}$ is the sum of the minimum hop counts corresponding to all secondary substations. Another variable $l_{i}$ denotes the number of secondary substations connected with primary substation $i$. It can also be called the link degree of primary substation $i$. The sum $L_{P(j)}$ of all link degrees corresponding to all primary 
substations in $P(j)$ is the link degree sum. In this example, the $l_{4}$ corresponding to B4 is 6 , and $L_{[\mathrm{B} 4, \mathrm{~B} 10, \mathrm{~B} 13]}$ is 17 .

The smaller $S_{P(j)}$ is, the better the $P(j)$ is. On the contrary, the bigger $L_{P(j)}$ is, the better the $P(j)$ is. Therefore, the reciprocal of $L_{P(j)}$ should be chosen to build the minimum objective function $Q_{P(j)} . S_{P(j)}$ and $1 / L_{P(j)}$ should be normalized before the function is built because their dimensions are different. Therefore, for a region system having $n$ substations whose $n_{p r i}$ is $m$, the model of the primary substation selection is shown in the following equations:

$$
\begin{gathered}
Q_{P(j)}=\min _{j=1}^{C_{m}^{n}}\left\{\omega_{1} \times S_{P(j)}{ }^{1}+\omega_{2} \times\left(1 / L_{P(j)}\right)^{1}\right\} \\
s_{i}=\min \left\{J_{1}, J_{2} \ldots J_{m}\right\} \\
S_{P(j)}=\sum_{i=1}^{n-m} s_{i} \\
S_{P(j)}{ }^{1}=\frac{S_{P(j)}-S_{\min }}{S_{\max }-S_{\min }} \\
L_{P(j)}=\sum_{i=1}^{m} l_{i} \\
\left(1 / L_{P(j)}\right)^{1}=\frac{\left(1 / L_{P(j)}\right)-(1 / L)_{\min }}{(1 / L)_{\max }-(1 / L)_{\min }}
\end{gathered}
$$

In Equation (4), for the $j$ th primary substation combination $P(j), S_{P(j)}{ }^{1}$ and $\left(1 / L_{j}\right)^{1}$ are the minimum hops sum and link degree sum after normalizing. The weights $\omega_{1}$ and $\omega_{2}$ are determined by the analytic hierarchy process (AHP) [15]. In Equation (5), $s_{i}$ is the minimum minimum hop count, as introduced with the example in Figure 2, of secondary substation $i . J_{1}, J_{2}, \ldots, J_{m}$ are the minimum hop counts between the secondary substation $i$ and the $m$ primary substations. $s_{i}$ is the minimum of $J_{1}, J_{2}, \ldots, J_{m}$. The sum of the $s_{i}$ values is calculated in Equation (6) and $S_{P(j)}$ is normalized in Equation (7). $S_{\max }$ and $S_{\min }$ are the maximum and minimum of $S_{P(j)}$. In Equation (8), for the $j$ th primary substation combination $P(j), l_{i}$ is the link degree of the $i$ th primary substation, and $L_{P(j)}$ is the sum of all $l_{i} . L_{j}$ is normalized in Equation (9). $(1 / L)_{\max }$ and $(1 / L)_{\min }$ are the maximum and minimum of $1 / L_{j}$, respectively.

The primary substation combination is the best one when $Q_{j}$ is at a minimum. Then, the primary substations in the combination are chosen.

\subsection{Secondary Substation Partition Model}

The delay time of data transmission mainly consists of transmission delay time, propagation delay time, queuing delay time, and processing delay time. The transmission delay time is related to the channel bandwidth, the propagation delay time is related to the channel length, the processing delay time is related to the number of hops through the data transmission, and the queuing delay time is related to the channel utilization [18]. The chosen partition affects the communication index of the secondary substation information transmission to the primary substation. In theory, the first strategy to realize protection is to reduce the delay time of information transmission. The second strategy is to ensure relative balance among the substations as well as the conformity of information.

The effects of transmission delay time, propagation delay time, and processing delay time on secondary substation partition are considered in this paper. The bigger the bandwidth is, the shorter the communication delay is. This means that we need choose a channel with large bandwidth. Therefore, we take the channel bandwidth's reciprocal $1 / b$, the weighted sum of the channel length $d$, and number of hops $J$ into consideration when building a model of secondary substation partitioning. It is necessary 
to normalize the three reference indices before weighted summation considering that the dimensions of the three reference indices are different. In summary, according to the influence factors of regional protection communication delay time, the secondary substation partition model is established as the following equations:

$$
q_{k}=\sum_{\substack{i=1 \\ k \in K}}^{i=t}\left(\omega_{3} \times\left(1 / b_{i}\right)^{1}+\omega_{4} \times d_{i}{ }^{1}\right)+\omega_{5} \times J^{1}
$$

$$
\begin{gathered}
\left(1 / b_{i}\right)^{1}=\frac{\left(1 / b_{i}\right)-(1 / b)_{\min }}{(1 / b)_{\max }-(1 / b)_{\min }} \\
d_{i}{ }^{1}=\frac{d_{i}-d_{\min }}{d_{\max }-d_{\min }} \\
J_{k}{ }^{1}=\frac{J_{k}}{J_{\max }} \\
M_{i} \leq 75 \% \times b_{i} .
\end{gathered}
$$

In the equations above,

- $K$ is the set of all possible paths between two substations;

- $\quad k$ is any path in the set;

- $\quad i$ is any channel of path $k^{\prime}$ s $t$ channels;

- $\omega_{3}, \omega_{4}$, and $\omega_{5}$ are the weights of the channel bandwidth, channel length, and the number of hops after normalization. The weight can be determined using the method in reference [23];

- $1 / b_{i}$ is the $i$ th channel's bandwidth reciprocal;

- $\left(1 / b_{i}\right)^{1}$ is the normalized value of the $i$ th channel's bandwidth reciprocal;

- $(1 / b)_{\max }$ and $(1 / b)_{\min }$ are the maximum and minimum values, respectively, of the channel bandwidth reciprocal for the entire communication network;

- $\quad d_{i}$ is the length of the $i$ th channel;

- $d_{i}^{1}$ is the normalized value of the $i$ th channel's length;

- $\quad d_{\max }$ and $d_{\min }$ are the maximum and minimum channel lengths, respectively, for the entire communication network.

The data transmission of the communication system usually has a certain hop limit in order to ensure that the communication delay time is within the scope of the regional protection. The communication network allows the maximum number of hops to be $J_{\max }$, usually 5 hops [18]. $J_{k}$ is the number of hops the path $\mathrm{k}$ has passed, and $J_{k}{ }^{1}$ is the total hops of the path $k$ after normalization. A constraint for ensuring information integrity is proposed in Equation (14), where $M_{i}$ is the traffic on the $i$ th channel. It is necessary to check whether the traffic on each channel meets the constraint condition, and if not, return to the primary substation selection step.

For path $k$ between two substations, the number of channels $t$ is equal to the number of hops $J_{k}$. Therefore, Equation (10) is equal to Equation (15).

$$
\begin{aligned}
q_{k} & =\sum_{\substack{i=1 \\
k \in K}}^{t}\left(\omega_{3} \times\left(1 / b_{i}\right)^{1}+\omega_{4} \times d_{i}{ }^{1}+\omega_{5} \times\left(1 / J_{\max }\right)\right)-\omega_{5} \times\left(1 / J_{\max } \times t\right)+\omega_{5} \times J^{1} \\
= & \sum_{i=1}^{t}\left(\omega_{3} \times\left(1 / b_{i}\right)^{1}+\omega_{4} \times d_{i}{ }^{1}+\omega_{5} \times\left(1 / J_{\max }\right)\right)
\end{aligned}
$$

To find the minimum $q_{k}$ of two substations more easily, a variable $Q(g, h)$ should be built as in Equation (16), based on Equation (15) by means of graph theory. The $Q(g, h)$ can reflect 
the communication delay of the channel between $g$ and $h$, so we define it as the communication distance. The bandwidth matrix $\boldsymbol{B}$ and length matrix $\boldsymbol{D}$ can be built using Equations (17) and (18). Then, the minimum $q_{k}$ can be calculated easily by using the Floyd-Warshall algorithm based on the communication distance matrix $Q$ including all $Q(g, h)$.

$$
\boldsymbol{Q}(g, h)= \begin{cases}\omega_{3} \times(1 / \boldsymbol{B}(g, h))^{1}+\omega_{4} \times \boldsymbol{D}(g, h)^{1}+\omega_{5} \times \boldsymbol{A}(g, h) / J_{\max }, & g \text { and } h \text { are directly connected } \\ \text { Inf }, & g \text { and } h \text { are not directly connected } \\ 0, & g=h\end{cases}
$$

$$
\begin{gathered}
\boldsymbol{B}(g, h)= \begin{cases}b_{g h}, & g \text { and } h \text { are directly connected } \\
\text { inf }, & g \text { and } h \text { are not directly connected } \\
0, & g=h\end{cases} \\
\boldsymbol{D}(g, h)= \begin{cases}d_{g h,} & g \text { and } h \text { are directly connected } \\
\text { inf }, & g \text { and } h \text { are not directly connected } \\
0, & g=h\end{cases}
\end{gathered}
$$

In the above equations, $b_{g h}$ and $d_{g h}$ are the channel bandwidth and channel length, respectively, between substations $g$ and $h$. The calculations of $(1 / \boldsymbol{B}(g, h))^{1}$ and $\boldsymbol{D}(g, h)^{1}$ are similar to the methods for $\left(1 / b_{i}\right)^{1}$ and $d_{i}^{1}$ in Equations (11) and (12).

The matrix $Q_{k}$ including all minimum $q_{k}$ between two substations can be created by using the Floyd-Warshall algorithm based on the communication distance matrix $Q$. Each secondary substation can find its optimal primary substation according to the matrix $Q_{k}$.

\subsection{Partition Correction}

\subsubsection{Partition Correction under an N-1 Channel Fault}

If an N-1 channel fault occurs after the partitioning, it is necessary to modify the partition according to the different channel fault situations.

Principle 1: When there are circuitous paths in the region, the information on the broken channel is circuitous through the circuitous path of the region. The partition need not be changed in this case.

Principle 2: If there is no circuitous path in the area, the information on the broken channel needs to be transmitted to other regions for circuitousness. It is judged whether the delay time meets the request if the information on the broken channel is circuitous with the help of channels in other regions. If satisfied, it is bypassed by other areas and the secondary substation remains unchanged; if not, the information is transmitted to the primary substation with the smallest delay time, and the secondary substation is partitioned into the corresponding new region.

The above principles are explained in conjunction with the system shown in Figure 3. Area 1 includes secondary substations B1, B2, and B3, and the primary substation is B3. Area 2 includes secondary substations B4, B5, B6, and B7, and the primary substation is B5.

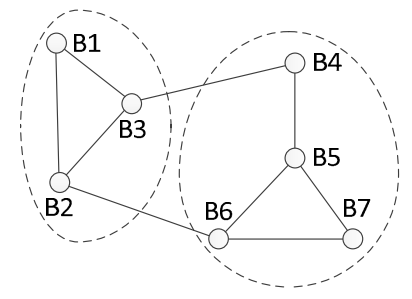

Figure 3. The system with seven substations in two regions, which can be used to explain partition correction under an N-1 channel fault.

According to Principle 1, circuitousness should be made through channel B7-B6-B5 when the channel between B7 and B5 is interrupted. This is shown in Figure 4a. When the channel between B4 
and B5 is interrupted, according to Principle 2, it should be determined whether the communication delay time constraint is satisfied by the circuitousness through channel B4-B3-B2-B6-B5. If satisfied, as shown in Figure $4 \mathrm{~b}$, circuitousness is carried out through this circuitous channel. If not, as shown in Figure 4c, secondary substation B4 will transmit the information to the primary substation B3 of Area 1.

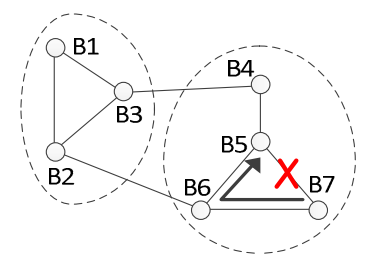

(a)

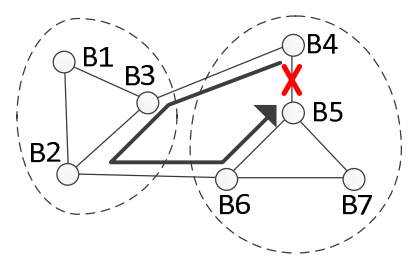

(b)

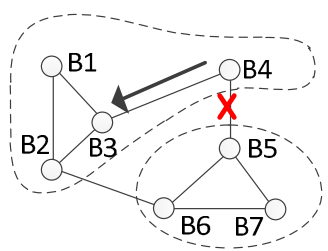

(c)

Figure 4. Corrections under different situations: (a) The circuitous process of information when it is acceptable to circuit within its own region; (b) The circuitous process of information when the help of other regions is necessary to circuit; (c) The partition correction when the delay will be too long if circuiting within its own region or with the help of other regions.

\subsubsection{Primary Substation Correction for Single-Point Failure}

After completing the selection of the primary substation, the regional protection configuration must consider setting a dual or even triple primary substation decision unit according to the importance of the protection object. When the primary substation has serious faults such as a DC fault, it is necessary to set up other secondary substations in the area as the backup primary substation. The principle of selecting the backup primary substation is based on the connection degree of each secondary substation in the region. The secondary substation with the highest connection degree is selected as the backup primary substation. When the communication between the local secondary substation and the primary substation is interrupted, the backup primary substation will automatically switch to the primary substation to realize the function of regional protection, so as to deal with the single-point failure risk of the system.

\section{Partition Process}

There are 39 points in the IEEE39 node system. Each point corresponds to a bus bar. However, the multisource information is transmitted between substations. The points of the communication network should correspond to substations; therefore, bus bars in the same substation should be merged into one point. The IEEE39 node system's communication network after merging is as shown in Figure 5 [24]. The partition strategy is explained using this network in the subsequent processes. The length and bandwidth of each channel of this system are shown in Table A1.

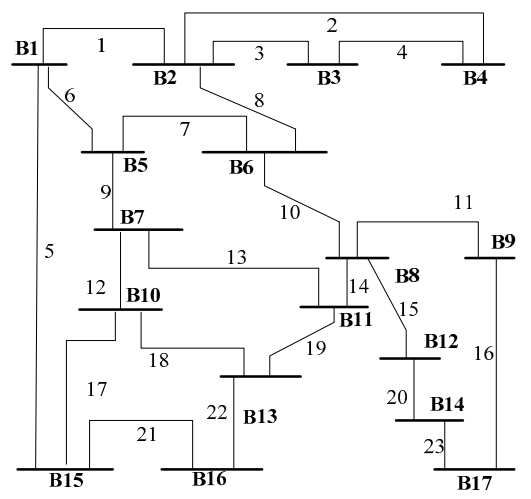

Figure 5. The IEEE39 node system's communication network after merging bus bars in the same substation as one point, which is convenient for introducing the partition strategy proposed in this article. 
The partitioning steps are as follows:

(1) The number of primary substation combinations can be determined according to Equation (1). It is 3 in this example. There are $C_{3}^{17}$ kinds of primary substation combinations, $P(1), P(2), \ldots$, $P\left(C_{3}^{17}\right)$, according to Equation (2). The collection $P$ of them is shown below.

$$
\boldsymbol{P}=\{[1,2,3],[1,2,4], \cdots,[15,16,17]\}
$$

(2) The connection matrix $A$ can be obtained by using Equation (3) considering the topology of communication system.

$$
A=\left[\begin{array}{ccccc}
0 & 1 & \cdots & \operatorname{Inf} & \operatorname{Inf} \\
1 & 0 & & & \operatorname{Inf} \\
\vdots & & \ddots & & \vdots \\
\operatorname{Inf} & & & 0 & \operatorname{Inf} \\
\operatorname{Inf} & \operatorname{Inf} & \cdots & \operatorname{Inf} & 0
\end{array}\right]
$$

(3) The optimal primary substation combination $\boldsymbol{P}(j)$ corresponding to the minimum $\boldsymbol{Q}_{P(j)}$ can be found from $\boldsymbol{P}$ according to Equations (3)-(8). It is $\boldsymbol{P}(j)=[2,8,10]$ in this example. The primary substations chosen are B2, B8, and B10.

(4) The bandwidth matrix $\boldsymbol{B}$, length matrix $\boldsymbol{D}$, and connection matrix $A$ can be determined according to Equations (16)-(18). They are shown below.

$$
\begin{aligned}
& \boldsymbol{B}=\left[\begin{array}{ccccc}
0 & 100 & \cdots & \operatorname{Inf} & \text { Inf } \\
100 & 0 & & & \text { Inf } \\
\vdots & & \ddots & & \vdots \\
\text { Inf } & & & 0 & \text { Inf } \\
\text { Inf } & \text { Inf } & \cdots & \text { Inf } & 0
\end{array}\right] \\
& \boldsymbol{D}=\left[\begin{array}{ccccc}
0 & 64.6 & \cdots & \text { Inf } & \text { Inf } \\
64.6 & 0 & & & \text { Inf } \\
\vdots & & \ddots & & \vdots \\
\operatorname{Inf} & & & 0 & \operatorname{Inf} \\
\operatorname{Inf} & \text { Inf } & \cdots & \text { Inf } & 0
\end{array}\right]
\end{aligned}
$$

(5) In this system, $\omega_{3}, \omega_{4}$, and $\omega_{5}$ can be determined as $0.35,0.15$, and 0.5 by the method in reference [15]. The weight matrix $Q$ can be calculated using Equation (15) based on matrices $\boldsymbol{A}, \boldsymbol{B}$ and $\boldsymbol{D}$.

$$
Q=\left[\begin{array}{ccccc}
0 & 0.39 & \cdots & \text { Inf } & \text { Inf } \\
0.39 & 0 & & & \text { Inf } \\
\vdots & & \ddots & & \vdots \\
\operatorname{Inf} & & & 0 & \operatorname{Inf} \\
\text { Inf } & \text { Inf } & \cdots & \text { Inf } & 0
\end{array}\right]
$$

(6) The optimal path weight $q_{g h}$ between any two substations is found. The matrix $Q_{k}$ including them all can be obtained by the Floyd-Warshall algorithm on the basis of Equations (10)-(18).

$$
Q_{k}=\left[\begin{array}{cccc}
0 & 0.39 & \cdots & 1.86 \\
0.39 & 0 & & \vdots \\
\vdots & & \ddots & 2.18 \\
1.86 & \cdots & 2.18 & 0
\end{array}\right]
$$


(7) The secondary substations can be divided into the suitable regions according to the primary substations chosen in Step (3), the $Q_{k}$ calculated in Step (6), and the planned number of secondary substations. The preliminary partition results are shown in Table 1.

Table 1. Results of partitioning by planning number.

\begin{tabular}{cc}
\hline Primary Substation & Secondary Substation \\
\hline B2 & B1 B3 B4 B6 B15 \\
B8 & B5 B6 B9 B12 B17 \\
B10 & B1 B7 B11 B13 B15 \\
\hline
\end{tabular}

(8) Whether there are unallocated secondary substations should be judged. If so, jump to Step (9); if not, jump to Step (10). In this example, the secondary substations B14 and B16 are not partitioned. So, we should jump to Step (9).

(9) For each secondary substation not assigned to a partition, the weight set between the remaining secondary substations and the primary substations can be obtained according to the $Q_{k}$ in Step (5). The primary substation corresponding to the minimum weight is the optimal primary substation of the secondary substation. Then, all unassigned substations to the optimal primary substations can be assigned similarly. In this example, the weights of the remaining secondary substations between the primary substations are obtained as follows.

$$
\begin{aligned}
& Q(\mathrm{~B} 14)=\left[\begin{array}{lll}
1.77 & 0.89 & 2.21
\end{array}\right] \\
& Q(\mathrm{~B} 16)=\left[\begin{array}{lll}
1.33 & 1.38 & 0.79
\end{array}\right]
\end{aligned}
$$

The weight between B14 and B8 is the minimum at 0.89, and the weight between B16 and $\mathrm{B} 10$ is the minimum at 0.79 . Therefore, the secondary substation B14 should be assigned to the corresponding region of the primary substation B8, and B16 should be assigned to the corresponding region of the primary substation B10.

(10) The repeated secondary substations of different regions are configured as boundary secondary substations. Then, the partition results are corrected according to the principle of partition correction under N-1 channel fault. The partition results can then be output using the MATLAB program written in accordance with the theory presented in this paper. The partition results of this example are as Table 2.

Table 2. Results after the uniform distribution of secondary substations.

\begin{tabular}{cc}
\hline Primary Substation & Secondary Substation \\
\hline B2 & B1 B3 B4 B6 B15 \\
B8 & B5 B6 B9 B12 B14 B17 \\
B10 & B1 B7 B11 B13 B15 B16 \\
\hline
\end{tabular}

In summary, the processes of the regional protection partition strategy considering all communication constraints, such as the lengths and bandwidths of the channels, are shown in Figure A1.

\section{Example Analysis}

In order to further verify the effectiveness of the partition strategy in this paper, the communication system of the $220 \mathrm{kV}$ power network in East Hubei, China (Figure A2), was partitioned using the partition strategy. The channel length, channel bandwidth, and other parameters of this system are shown in Table A2. 
According to Equation (8), it is determined that the preliminary number of primary substations is 8. In order to give theoretical guidance and partitioning advice to the actual engineering demand, the number of regions was set to be 7,8 , and 9 . The partition results are shown in Tables 3-5.

Table 3. Results of dividing into 7 regions.

\begin{tabular}{ccc}
\hline Region & Primary Substation & Secondary Substation \\
\hline 1 & Fenghuang & Guanshan, Yuefu, Luojia, Ye, Xunsi, Dian, Miaoshan, Shatang \\
2 & Wangzhuang & Chaan, Lumen, Puqi, Xianning, Tangjiao, Wutian \\
3 & Qiru & Cihu, Xialu, Sike, Jiangiia, Tieshan, Xiangjia \\
4 & He & Shahu, Beiyang, Qingshan, Ya, Wudong, Gang, Huashan \\
5 & Langjia & Lian, Tieshan, Xialu, Ezhou, Huarong, Zuoling \\
6 & Qichun & Caicheng, Cuijia, Zhangjia, Xisai \\
7 & Shiban & Hangshi, Xisai, Sike, Weiyuan, Cihu, Daji \\
\hline
\end{tabular}

Table 4. Results of dividing into 8 regions.

\begin{tabular}{ccc}
\hline Region & Primary Substation & Secondary Substation \\
\hline 1 & Fenghuang & Guanshan, Yuefu, Luojia, Ye, Xunsi, Dian, Miaoshan, Shatang \\
2 & Wangzhuang & Chaan, Lumen, Puqi, Xianning, Tangjiao, Wutian \\
3 & Qiru & Cihu, Xialu, Sike, Jiangjia, Tieshan, Xiangjia \\
4 & He & Shahu, Beiyang, Qingshan, Ya, Wudong \\
5 & Langjia & Lian, Tieshan, Xialu, Ezhou, Huarong \\
6 & Qichun & Caicheng, Cuijia, Zhangia, Xisai \\
7 & Shiban & Huangshi, Xisai, Sike, Weiyuan, Daji \\
8 & Huashan & Wudong, Gang, Ya, Ezhou, Beiyang, Zuoling \\
\hline
\end{tabular}

Table 5. Results of dividing into 9 regions.

\begin{tabular}{ccc}
\hline Region & Primary Substation & Secondary Substation \\
\hline 1 & Fenghuang & Yuefu, Luojia, Ye, Dian, Miaoshan, Shatang \\
2 & Wangzhuang & Chaan, Lumen, Puqi, Xianning, Tangjiao, Wutian \\
3 & Qiru & Cihu, Xialu, Sike, Jiangjia, Xiangjia \\
4 & He & Shahu, Beiyang, Qingshan, Ya \\
5 & Langiia & Lian, Tieshan, Xialu, Ezhou, Huarong \\
6 & Qichun & Caicheng, Cuijia, Zhangjia \\
7 & Shiban & Huangshi, Xisai, Sike, Weiyuan, Daji \\
8 & Huashan & Wudong, Gang, Ya, Ezhou, Zuoling \\
9 & Guanshan & Xunsi, Ya, Qingshan, Gang \\
\hline
\end{tabular}

For the partitioning results in Tables $3-5$, we need to determine whether the longest delay time in each region meets the rapidity requirement of the regional protection communication, and determine whether there is channel jam in each region. We can thereby determine whether the partition results meet the regional protection partitioning balance requirements. According to reference $[25,26]$, the size of the data uploaded by each substation was set to be $21.7 \mathrm{Mbps}$. The calculation method for communication delay time is referenced in [24]. The communication indicator results of the partition are shown in Table 6.

Some conclusions can be obtained from the results in Table 6. The delay time will be longer when the number of partitions is fewer, and the more serious problem is that the channel utilization rate exceeds $50 \%$. The channel delay time will increase nonlinearly in this case, as discussed in reference [27]. In addition, the amount of information will sharply increase when the fault occurs; thus, it will be difficult to meet the needs of regional protection. With the increase of the number of regions, the delay time of each region is obviously reduced, and the load amount tends to be more uniform. However, it also means that there are more primary substations which means higher investment costs. Therefore, according to the actual requirements of the project, considering the real-time, equilibrium, and investment economy of the system, the appropriate number of regions can be determined. For example, this system adopts 8 regions in the partition. 
Table 6. Result contradistinction of communication indicators.

\begin{tabular}{ccccccc}
\hline \multirow{2}{*}{ Region } & \multicolumn{2}{c}{$\begin{array}{c}\text { Longest Delay Time with Different } \\
\text { Numbers of Partitions (ms) }\end{array}$} & \multicolumn{3}{c}{$\begin{array}{c}\text { Biggest } \\
\text { Different }\end{array}$} & $\begin{array}{c}\text { Chnel Utilization with } \\
\text { Numbers of Partitions }\end{array}$ \\
\hline & 7 Regions & 8 Regions & 9 Regions & 7 Regions & 8 Regions & 9 Regions \\
\hline 1 & 2.55 & 2.55 & 2.43 & $28 \%$ & $28 \%$ & $28 \%$ \\
2 & 2.49 & 2.49 & 2.49 & $28 \%$ & $28 \%$ & $28 \%$ \\
3 & 3.37 & 3.37 & 3.37 & $42 \%$ & $42 \%$ & $28 \%$ \\
4 & 3.79 & 2.65 & 2.63 & $70 \%$ & $42 \%$ & $28 \%$ \\
5 & 3.73 & 2.52 & 2.52 & $42 \%$ & $28 \%$ & $28 \%$ \\
6 & 3.06 & 3.06 & 1.90 & $28 \%$ & $28 \%$ & $14 \%$ \\
7 & 2.60 & 2.30 & 2.30 & $28 \%$ & $28 \%$ & $28 \%$ \\
8 & - & 2.53 & 2.53 & - & $42 \%$ & $28 \%$ \\
9 & - & - & 2.40 & - & - & $28 \%$ \\
\hline
\end{tabular}

The communication indicators of the system partitioned by the traditional strategy from reference [28] can be acquired using the calculation method in reference [24]. The result comparison of partitioning using different strategies is shown in Table 7.

Table 7. Result contradistinction of partitioning using different strategies.

\begin{tabular}{ccccccc}
\hline Strategy & \multicolumn{3}{c}{ Longest Delay Time (ms) } & \multicolumn{3}{c}{ Biggest Channel Utilization } \\
\hline Strategy from this article & $\begin{array}{c}3.79 \\
\text { (7 regions) }\end{array}$ & $\begin{array}{c}3.37 \\
\text { (8 regions) }\end{array}$ & $\begin{array}{c}3.37 \\
\text { (9 regions) }\end{array}$ & $\begin{array}{c}70 \% \\
\text { (7 regions) }\end{array}$ & $\begin{array}{c}42 \% \\
\text { (8 regions) }\end{array}$ & $\begin{array}{c}28 \% \\
\text { (9 regions) }\end{array}$ \\
\hline $\begin{array}{c}\text { Traditional strategy from } \\
\text { reference [28] }\end{array}$ & \multicolumn{3}{c}{$81.7 \%$} \\
\hline
\end{tabular}

The system partitioned according to the strategy from this article performs better than that partitioned according to the traditional strategy from reference [28] in terms of communication delay and channel utilization. The most important reason for this is that only the number of hops and the topological relation are considered in reference [28]. Therefore, the partition of Region 1 is obviously unbalanced, and the transmission delay times of Region 1 and Region 3 are a little long. In this article, other factors affecting the transmission delay time such as channel distance and bandwidth are considered. A better partition can be provided by using the strategy introduced in this article.

\section{Conclusions}

Regional protection can solve the problems associated with traditional protection. However, it needs a lot of multisource information, so it is important to choose a suitable partition strategy. The in situ methods of partitioning focus only on the effect of hops. The effects of distance and bandwidth were also dealt with in this article. In this article, a primary substation selection model and a secondary substation partition model were established based on the regional protection communication constraints. Next, methods of corrections for $\mathrm{N}-1$ channel faults and single-point failure were illustrated. Programs based on the strategy were written in MATLAB, meaning that a large number of artificial judgments and assignments of traditional strategy are not required. Finally, a comparative analysis of several partitions was conducted. Suggestions on how to choose the most suitable partition considering economy and the communication delay were given. All data can be quickly dealt with by MATLAB using the strategy from this article, and several good partitions and suggestions can be given.

Author Contributions: Conceptualization, Y.G. and Z.L.; Data curation, Y.G.; Formal analysis, Y.G. and Z.L.; Funding acquisition, Z.L.; Methodology, Y.G. and Z.L.; Resources, Z.L.; Supervision, H.T. and P.W.; Writing_original draft, Y.G.; and Writing_review and editing, Y.G., L.W., H.T. and P.L.K.

Funding: This research was funded by National Natural Science Foundation of China (No. 51607106) and key projects of the science and technology research program of the Hubei Provincial Education Department (No. Z2017037).

Conflicts of Interest: The authors declare no conflicts of interest. 


\section{Appendix A}

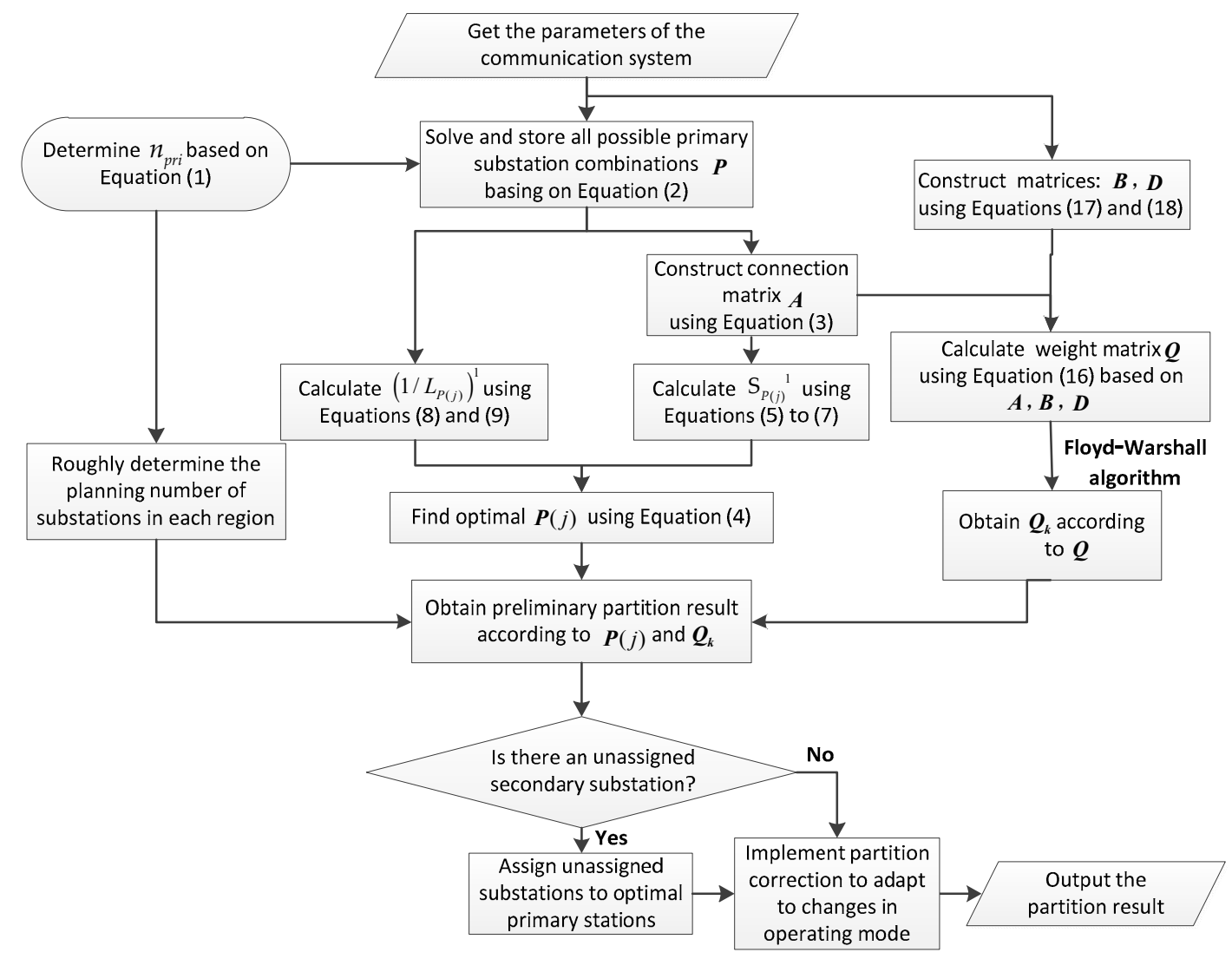

Figure A1. The processes of the regional protection partition strategy considering all communication constraints, such as the lengths and bandwidths of channels.

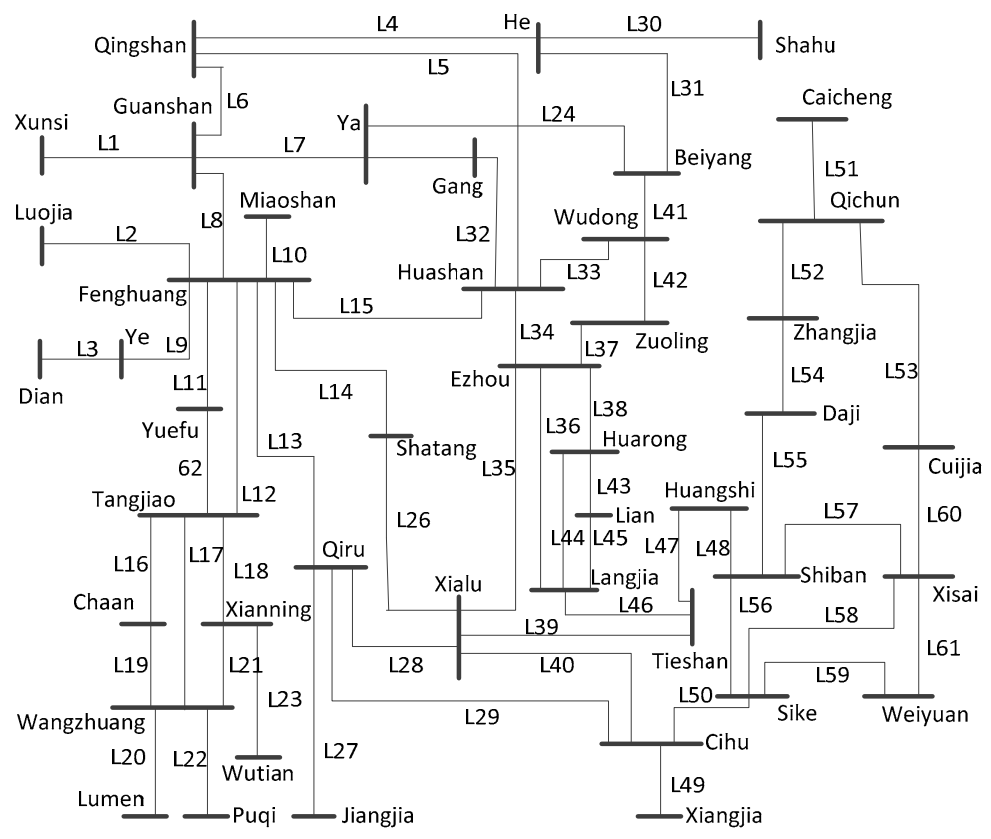

Figure A2. The $220 \mathrm{kV}$ grid communication system in the eastern region of Hubei province, China, to verify the applicability of the partition strategy proposed in this article under larger system conditions. 
Table A1. Communication system data of IEEE39.

\begin{tabular}{ccccccccccccc}
\hline Channel & $\mathbf{1}$ & $\mathbf{2}$ & $\mathbf{3}$ & $\mathbf{4}$ & $\mathbf{5}$ & $\mathbf{6}$ & $\mathbf{7}$ & $\mathbf{8}$ & $\mathbf{9}$ & $\mathbf{1 0}$ & $\mathbf{1 1}$ & $\mathbf{1 2}$ \\
\hline Length $(\mathrm{km})$ & 64.6 & 125 & 94.8 & 30.2 & 127.4 & 30.2 & 43 & 34.6 & 42.6 & 17.8 & 27 & 25.6 \\
Bandwidth (Mbit) & 100 & 100 & 100 & 50 & 50 & 50 & 100 & 100 & 50 & 100 & 100 & 100 \\
\hline Channel & $\mathbf{1 3}$ & $\mathbf{1 4}$ & $\mathbf{1 5}$ & $\mathbf{1 6}$ & $\mathbf{1 7}$ & $\mathbf{1 8}$ & $\mathbf{1 9}$ & $\mathbf{2 0}$ & $\mathbf{2 1}$ & $\mathbf{2 2}$ & $\mathbf{2 3}$ \\
\hline Length (km) & 69.2 & 18.8 & 11.8 & 28 & 22.4 & 25.8 & 43.4 & 70 & 27.6 & 20.2 & 19.2 \\
Bandwidth (Mbit) & 100 & 100 & 100 & 100 & 100 & 100 & 100 & 100 & 50 & 50 & 50 \\
\hline
\end{tabular}

Table A2. The communication system parameters of the $220 \mathrm{kV}$ grid in the eastern region of Hubei, China.

\begin{tabular}{cccccccccccccc}
\hline Channel & L1 & L2 & L3 & L4 & L5 & L6 & L7 & L8 & L9 & L10 & L11 & L12 & L13 \\
\hline Length $(\mathrm{km})$ & 14 & 20 & 8 & 34.6 & 63.4 & 31 & 22 & 31 & 25 & 50 & 18 & 83 & 87 \\
Bandwidth (Mbit) & 155 & 155 & 155 & 155 & 155 & 155 & 155 & 155 & 155 & 155 & 155 & 155 & 622 \\
\hline Channel & L14 & L15 & L16 & L17 & L18 & L19 & L20 & L21 & L22 & L23 & L24 & L25 & L26 \\
\hline Length $(\mathrm{km})$ & 116 & 104 & 79 & 52 & 17 & 18 & 25 & 43 & 40 & 34 & 23 & 8 & 43 \\
Bandwidth (Mbit) & 155 & $\mathbf{6 2 2}$ & 155 & 155 & 155 & 155 & 155 & 155 & 155 & 155 & 155 & 155 & 155 \\
\hline Channel & L27 & L28 & L29 & L30 & L31 & L32 & L33 & L34 & L35 & L36 & L37 & L38 & L39 \\
\hline Length $(\mathrm{km})$ & 40.5 & 34 & 23 & 23 & 30 & 17.4 & 14 & 39 & 55 & 32 & 21 & 8.4 & 9 \\
Bandwidth (Mbit) & 155 & 155 & 155 & 155 & 155 & 155 & 155 & 155 & 155 & 155 & 155 & 155 & 155 \\
\hline Channel & L40 & L41 & L42 & L43 & L44 & L45 & L46 & L47 & L48 & L49 & L50 & L51 & L52 \\
\hline Length $(\mathrm{km})$ & 11 & 25 & 29 & 27 & 34 & 9 & 14 & 41 & 9 & 42 & 8 & 62 & 80 \\
Bandwidth (Mbit) & 155 & 155 & 155 & 155 & 155 & 155 & 155 & 155 & 155 & 155 & 155 & 155 & 155 \\
\hline Channel & L53 & L54 & L55 & L56 & L57 & L58 & L59 & L60 & L61 & L62 & & & \\
Length $(k m)$ & 75 & 44 & 36 & 16.5 & 9 & 25 & 18 & 21 & 10.5 & 62 & & & \\
Bandwidth (Mbit) & 155 & 155 & 155 & 155 & 155 & 155 & 155 & 155 & 155 & 155 & & & \\
\hline
\end{tabular}

\section{References}

1. Wang, Z.; He, J.; Xu, Y.; Crossley, P. Multi-objective optimisation method of power grid partitioning for wide-area backup protection. IET Gener. Transm. Distrib. 2018, 12, 696-703. [CrossRef]

2. Jena, M.K.; Samantaray, S.R.; Panigrahi, B.K. A New Wide-Area Backup Protection Scheme for Series-Compensated Transmission System. IEEE Syst. J. 2017, 11, 1877-1887. [CrossRef]

3. Lan, T.; Li, Y.; Duan, X. Simplified Analytic Approach of Pole-to-Pole Faults in MMC-HVDC for AC System Backup Protection Setting Calculation. Energies 2018, 11, 264. [CrossRef]

4. Li, Z.; Meng, X.; Wang, X. A wide-area relaying protection system based on multi-point measurement information. IEEJ Trans. Electr. Electron. Eng. 2018, 13, 529-536. [CrossRef]

5. Wang, F.; Yu, M.; Bo, Z. Substation area joint defensive protection strategy based on distributed cooperative all-in-one device. J. Modern Power Syst. Clean Energy 2018, 3, 467-477.

6. Osman, A.; Hassan, M.; Sulaiman, M. Communication-based Adaptive Protection for Distribution Systems Penetrated with Distributed Generators. Power Syst. Protect. Control 2012, 40, 1-9. [CrossRef]

7. Zare, J.; Aminifar, F.; Sanaye-Pasand, M. Communication-Constrained Regionalization of Power Systems for Synchrophasor-Based Wide-Area Backup Protection Scheme. IEEE Trans. Smart Grid 2015, 6, 1530-1538. [CrossRef]

8. Ivankovic, I.; Kuzle, I.; Holjevac, N. Wide Area Information-Based Transmission System Centralized Out-of-Step Protection Scheme. Energies 2017, 10, 633. [CrossRef]

9. Li, Z.; Yin, X.; Zhang, Z. Wide-Area Protection Fault Identification Algorithm Based on Multi-Information Fusion. IEEE Trans. Power Deliv. 2013, 28, 1348-1355.

10. Appasani, B.; Mohanta, D.K. Co-Optimal Placement of PMUs and Their Communication Infrastructure for Minimization of Propagation Delay in the WAMS. IEEE Trans. Ind. Inform. 2018, 14, 2120-2132. [CrossRef]

11. Eissa, M.M. Developing wide area phase plane primary protection scheme "WA4PS" for complex smart grid system. Int. J. Electr. Power Energy Syst. 2018, 99, 203-213. [CrossRef]

12. Yin, X.G.; Wang, Y.; Zhang, Z. Zone-division and tripping strategy for limited wide area protection adapting to smart grid. Proc. Chin. Soc. Electr. Eng. 2010, 30, 1-7. 
13. Ma, J.; Wang, X.; Wang, Z. Partition of protection zone with circular overlapping coverage for wide-area protection system. Electr. Power Autom. Equip. 2012, 32, 50-54.

14. Jiang, X.; Wang, Z.; Li, C. Zoning scheme of regional autonomy backup protection. Trans. China Electrotech. Soc. 2013, 28, 234-241.

15. He, Z.; Zhang, Z.; Yin, X. Partition model and optimization algorithm for wide-area backup protection based on centralized decision-making mode. Trans. China Electrotech. Soc. 2014, 29, 212-219.

16. Kong, D.; Lv, F. Wide-area backup protection algorithm based on regional multi-information fusion. Power Syst. Protect. Control 2017, 4, 26-32.

17. He, J.; Wang, Z.; Zhang, D. Wide-area backup protection partitioning based on graph theory and fuzzy evaluation. Electr. Power Autom. Equip. 2017, 37, 75-82.

18. Drayer, E.; Kechagia, N.; Hegemann, J.; Braun, M.; Gabel, M. Distributed Self-Healing for Distribution Grids with Evolving Search Space. IEEE Trans. Power Deliv. 2018, 33, 1755-1764. [CrossRef]

19. Serizawa, Y.; Myoujin, M.; Kitamura, K. Wide-area current differential backup protection employing broadband communications and time transfer systems. IEEE Trans. Power Deliv. 1998, 13, 1046-1052. [CrossRef]

20. Wang, Z.P.; Zhang, Y.G.; Zhang, J.F. Recent Research Progress in Fault Analysis of Complex Electric Power Systems. Adv. Electr. Comput. Eng. 2010, 10, 28-33. [CrossRef]

21. Chen, L.X.; Chen, Z.; Huang, X.L.; Jin, L. A Study on Price-Based Charging Strategy for Electric Vehicles on Expressways. Energies 2016, 9, 385. [CrossRef]

22. Bielecki, W.; Kraska, K.; Klimek, T. Using basis dependence distance vectors in the modified Floyd-Warshall algorithm. J. Comb. Optim. 2015, 30, 253-275. [CrossRef]

23. Cale, J.L.; Johnson, B.B.; Dall'Anese, E.; Young, P.M. Mitigating Communication Delays in Remotely Connected Hardware-in-the-Loop Experiments. IEEE Trans. Ind. Electron. 2018, 12, 9739-9748. [CrossRef]

24. Xiong, X.; Tan, J.; Lin, X. Routing algorithm for communication system in wide-area protection based on MPLS. Trans. China Electrotech. Soc. 2013, 28, 257-263.

25. Dou, X.; Quan, X.; Wu, Z. Hybrid Multi-Agent Control in Microgrids: Framework, Models and Implementations Based on IEC 61850. Energies 2015, 8, 31-58. [CrossRef]

26. Short, M.; Abugchem, F.; Dawood, M. Tunneling Horizontal IEC 61850 Traffic through Audio Video Bridging Streams for Flexible Microgrid Control and Protection. Energies 2016, 9, 204. [CrossRef]

27. Marzal, S.; Gonzalez-Medina, R.; Salas-Puente, R. A Novel Locality Algorithm and Peer-to-Peer Communication Infrastructure for Optimizing Network Performance in Smart Microgrids. Energies 2017, $10,1275$.

28. Li, Z.; Yin, X.; Zhang, Z. Study on system architecture and fault identification of zone-division wide area protection. Proc. Chin. Soc. Electr. Eng. 2011, 31, 95-103. 